Tuberculosis Research Unit for the streptomycin trial, ${ }^{4}$ which subsequently (from 1948 ) served as a model for randomisation in many later randomised controlled trials? $\mathrm{Or}$ is it because the results of the patulin trial were negative and those of the streptomycin trial made medical history?

I have previously quoted Bradford Hill's recollection that his statistical, and my medical, experiences in this field converged when we prepared for the streptomycin project. ${ }^{56}$ The patulin trial certainly influenced my thinking. Yet, so far as I am aware, the literature on the modern clinical trial contains no recognition of this trial, despite its many deserving features. Should not this omission be rectified?

P D'ARCY HART Former director, MRC Tuberculosis Research Unit National Institute for Medical Research,

London NW7 1AA

1 Van der Wijden CL, Overbeke JA. Audits of reports of randomised clinical trials published in one journal over 45 years. BMF 1995;311:918. (7 October.)

2 Quoted in footnote to: D'Arcy Hart P. History of randomised control trials. Lancet 1972;i:965.

3 Medical Research Council. Clinical trial of patulin in the common cold. Lancet 1944; ii:373-5.

4 Medical Research Council. Streptomycin treatment of pulmonary tuberculosis. $B M$ F 1948;ii:769-83.

5 D'Arcy Hart P. Randomised controlled clinical trials. $B M F$ 1991;302:1271.

6 Bradford Hill A. Memories of the British streptomycin trial in tuberculosis. Control Clin Trials 1990;11:77-90.

\section{Recent advances in obstetrics}

\section{Testing for Down's syndrome carries too much stress}

EDrToR, - I would like to highlight some of the points that Philip Steer made about serum screening for Down's syndrome, ${ }^{1}$ in the light of personal experience.

My first reaction to the offer of an antenatal appointment to discuss a risk factor of 1 in 165 for Down's syndrome was one of surprise. I thought that this was too low a risk to contemplate amniocentesis. My second reaction was to consider the implications seriously for the first time and to realise my reluctance to go through with the late abortion of a fetus that might have the potential for a reasonably independent and fulfilled life. I thought it important, however, that my nonmedical husband should have more information, so we attended the clinic. Ironically, I missed a postgraduate course on antenatal screening in order to do so.

The visit was far from satisfactory. I was persuaded to have more detailed ultrasound scanning, despite having declined amniocentesis. The ultrasound scan showed the presence of chorion villus cysts, a marker for Down's syndrome but present in 3\% of normal fetuses. Suddenly the risk of Down's syndrome was increased, but in an unquantifiable way. The consultant now strongly recommended amniocentesis, even if only to prepare or reassure us, and enjoyment of my normal pregnancy was spoiled. I was anxious and confused. What in fact was the risk of Down's syndrome? My husband felt sure that he could not cope with a child with learning disabilities and preferred not to think about it, perhaps hoping that he would feel differently in the event if he had to. He respected my decision not to have a termination, and we both agreed to decline further testing.

Our anxiety persisted to the point of delivery and beyond. I was convinced for a few awful minutes, before reason intervened, that my perfectly normal baby (shocked after a rapid delivery) did have features of Down's syndrome. Now 2 years old, he is generally healthy but has had more than his fair share of medical problems. I wonder whether my experience in pregnancy had some affect on my ability to copy with his subsequent illnesses.

Serum screening has low sensitivity $(0-48 \%$ in the studies quoted), causes parents high levels of anxiety, puts normal fetuses at risk from amniocentesis, and is financially expensive. Routine use should be stopped, and adequate counselling of patients is obligatory.

DIANA WARNER
General practitioner

43 Nevil Road,

Bishopston,

Bristol BS7 9EG

1 Steer P. Recent advances in obstetrics. BMF 1995;311:1209-12. (4 November.)

\section{Figures on screening for Down's syndrome} are inaccurate

EDrToR,-Philip Steer's comments about serum screening for Down's syndrome ${ }^{1}$ should not go unchallenged. Firstly, it is inappropriate to cite a $48 \%$ detection rate. For a $5 \%$ false positive rate the estimated detection rate is $59 \%$ when dates are used to estimate gestational age and $65 \%$ if an estimate based on an ultrasound scan is used. ${ }^{2}$ The Barts Down's syndrome demonstration project, in which $48 \%$ of cases were detected, was not designed to estimate the detection rate and was not large enough to do so.

Secondly, a serum screening test carried out on a community basis costs much less than the $f 80$ cited. There are existing NHS programmes which are provided at a quarter of this sum.

Thirdly, contrary to Steer's statement, we know that screening has had a considerable effect on reducing the birth prevalence of Down's syndrome. In the absence of screening and at a time of increasing maternal age, the birth prevalence of Down's syndrome would have increased from $1.41 / 1000$ in 1989 to $1.47 / 1000$ in $1993,{ }^{3}$ assuming a natural fetal loss rate of $27 \%$ among the women who had terminations. In fact it has decreased from $1 \cdot 11 / 1000$ in 1989 to $0.92 / 1000$ in 1993 . This is equivalent to an increase in the percentage of affected births avoided through screening from about $21 \%$ to $37 \%$

Finally, it is acknowledged that the provision of adequate information before the screening test and appropriate counselling afterwards is important and is not always carried out satisfactorily. This is not the fault of the screening test but is a fault in the way the screening test is delivered to women. It underlines the fact that the test itself is but one component of the screening procedure.

ANNE KENNARD Lecturer EVA ALBERMAN Professor

Wolfson Institute of Preventive Medicine, Medical College of St Bartholomew's Hospital, London EC1M 6BQ

Brent and Harrow Health Authority,

Harrow,

Middlesex HA1 3EX

1 Steer P. Recent advances in obstetrics. BMf 1995;311:1209-12. (4 November.)

2 Wald NJ, Densem JW, Smith D, Klee GG. Four marker serum screening for Down's syndrome. Prenat Diagn 1994;14: 707-16.

3 Alberman E, Mutton D, Ide R, Nicholson A, Bobrow M. Down's syndrome births and pregnancy terminations in 1989 to 1993 : preliminary findings. Br f Obstet Gynaecol 1995;102:445-7.

\section{Epidural analgesia in labour}

EDITOR,-The comments on epidural analgesia for labour in Philip Steer's review overlooked important new evidence relating to postpartum backache and a potential hazard of ambulatory epidural analgesia.

Steer implies that the use of epidurals in labour results in increased rates of long term backache. However, the reference supporting this is a retrospective review of only $39 \%$ of a population of women who delivered their baby at a given hospital over an eight year period. ${ }^{2}$ Apart from the inherent drawbacks in making causal associations in a retrospective study, recall bias over such a protracted period would tend to render its conclusions unreliable.

On the contrary, a more recent prospective study of 1042 women interviewed during their admission for delivery and again two months later found no difference in the incidence of new postpartum back pain between those who received epidural analgesia for labour (44\%) and those who did not $(45 \%){ }^{3}$ The design of this study is more appropriate to assess cause and effect, and the results impressively refute the suggestion that epidural analgesia is a risk factor for "long term backache."

Furthermore, although Steer correctly states that the combined spinal-epidural technique of ambulatory labour analgesia may cause a greater degree of maternal hypotension than conventional epidural analgesia, he does not mention its potential impact on posterior column sensation, particularly proprioception. ${ }^{4}$ This may undermine safe ambulation, although there is no doubt that the ambulatory technique improves maternal satisfaction and that retention of lower limb mobility is an advance, even if actual walking may not be advisable.

DONAL J BUGGY

St Mary's Orthopaedic Hospital, Senior

Cappagh,

Republic of Ireland

1 Steer P. Recent advances in obstetrics. BMf 1995;311:1209-12. (4 November.)

2 MacArthur C, Lewis M, Knox EG, Crawford JS. Epidural anaesthesia and long term backache after childbirth. $B M$ 1990;301:9-12.

3 Breen TW, Ransil BJ, Groves PA, Oriol NE. Factors associated with back pain after childbirth. Anesthesiology 1994;81:29-34.

4 Buggy DJ, Hughes N, Gardiner J. Posterior column sensory impairment during ambulatory epidural analgesia in labour. $B$ f Anaesth 1994;73:540-2.

Transmission of HIV from mother to infant depends on many factors

EDITOR,-I was disturbed at the many unfounded assertions about preventing the transmission of HIV from mother to infant in Philip Steer's review of recent advances in obstetrics. ${ }^{1} \mathrm{He}$ asserts that transmission rates could be halved if women did not breast feed. This oversimplifies a very complicated reality, in which transmission to infants depends on many other factors as well; women who have HIV related illnesses may be at high risk of transmitting HIV to their infants whether they breast feed or not.

Secondly, Steer asserts that caesarean section reduces transmission by a "further $40 \%$." The evidence for this is far from clear cut, and the study cited does not draw this conclusion. No large controlled trials have ever been done, and no one has ever recommended routine caesarean section for HIV positive pregnant women on the basis of the existing evidence. Nor does Steer even consider that women with HIV might be at higher risk of complications from caesarean section, when there is at least anecdotal evidence that this is so. ${ }^{2}$

Thirdly, the role of zidovudine during pregnancy to prevent transmission to infants needs to be confirmed. One trial in one country is not enough. Just as the use of zidovudine in most developing countries for this purpose is highly unrealistic, given the cost, ${ }^{3}$ we might ask whether the NHS would pay for all pregnant women with HIV infection to use it. If not, there is no benefit.

Thus for Steer to reach the conclusion in a few sentences that, with these three (in his eyes) apparently simple precautions, vertical transmis- 